\title{
Crioablación laparoscópica de las pequeñas masas renales
}

\author{
M.P. Laguna Pes, B. Lagerveld, L.P.W. Witte, I. Kummerlin, H. Wijkstra, J.J.M.C.H. de la Rosette \\ Servicio de Urología. AMC Universidad de Amsterdam. Amsterdam. Holanda \\ Actas Urol Esp 2005; 29 (9): 860-868
}

\section{RESUMEN}

CRIOABLACIÓN LAPAROSCÓPICA DE LAS PEQUEÑAS MASAS RENALES

Objetivos: Revisión del estado actual de la crioblación de las pequeñas masas renales y descripción preliminar de la serie del AMC.

Material y Método: Búsqueda bibliográfica (PubMed/Medline/Embase) y análisis de las series más importantes. La presente serie incluye 13 pacientes portadores de masas renales únicas de pequeño tamaño tratados mediante crioablación laparoscópica con sondas ultrafinas (1,5 mm diámetro). El seguimiento postoperatorio se realizó mediante CT y/o RMI trimestralmente durante el primer año y semestralmente durante el segundo.

Resultados: No existen estudios randomizados comparando la crioablación de las masas renales de diámetro $\leq 4 \mathrm{~cm}$ con la cirugía parcial o radical. La tasa de complicaciones es baja y la tasa de recurrencia o persistencia, a corto plazo, mínima salvo en una serie de crioablación guiada por la imagen $(8 \%)$.

El diámetro tumoral máximo tratado en nuestra serie es de 3,2 cm. Los tumores se abordaron retro o transperitonealmente dependiendo de su situación. El tiempo quirúrgico medio fue de 208 minutos (106-379) y el tiempo medio de exposición a temperaturas inferiores a $-20^{\circ} \mathrm{C}$ en la periferia tumoral fue de 10 minutos. A tiempo medio de seguimiento de 8 meses no se objetivo recurrencia tumoral.

Conclusión: La crioablación laparoscópica o guiada mediante imagen de las masas renales de pequeño tamaño parece una alternativa aceptable si bien el tiempo medio de seguimiento es todavía corto en la mayoría de las series.

Palabras clave: Masa renal. Crioablación. Laparoscopia.

\section{ABSTRACT}

LAPAROSCOPIC ASSISTED CRYOABLATION OF SMALL RENAL MASSES

Aims: To review the current status of cryoablation of small renal masses and to preliminary report our experience at the AMC.

Material and methods: A bibliographic search was conducted (PubMed/Medline/Embase) and the most important series were analyzed. Our series includes 13 patients with a solitary small renal mass treated by Laparoscopic assisted Cryoblation with fine cryoprobes (1,5 cm diameter). Postoperative follow-up was done by means of CT and/or MRI every three months during the first year and every 6 months during the second year.

Results: There are no randomized trials comparing Cryoblation of renal masses $(<4 \mathrm{~cm}$ diameter $)$ with either radical surgery or partial nephrectomy but only case series. Complication rate is low as it is the recurrence or persistence rate in most of the series but one referring to radiologically guided Cryoablation (8\%).

The maximal diameter of the masses treated in our series were $3,2 \mathrm{~cm}$. Tumors were approached retro or transperitoneally depending on their localization in the kidney. Average surgical time were 208 minutes (108-379) and average time of exposition to temperatures lower than $-20^{\circ} \mathrm{C}$ in the tumor periphery was 10 minutes. AT a mean follow-up of 8 months no tumoral recurrence were objectivated.

Conclusions: Cryoablation of small renal masses may be an acceptable alternative of treatment although mean follow-up is still short in all the series. 
$\mathrm{L}$ a "American Cancer Society" calculaba en el año 2004, 32.000 nuevos diagnósticos de cáncer de riñón en USA, un tercio de los cuales en estadios localmente avanzado o metastático. Las muertes por cáncer de riñón se estimaban en 12.000. En ese mismo periodo el cáncer de riñón alcanzaría el séptimo lugar entre los cánceres del varón con una incidencia del 3\% y sería responsable del 3\% de las muertes por cáncer en el varón.

Datos de la SEER (Surveillance Epidemiology and End Results) demuestran que en la última década la incidencia del cáncer de riñón ha aumentado lenta pero constantemente a expensas del incremento en la tasa de tumores localizados pero también en la de tumores avanzados ${ }^{1}$.

Al margen de otras causas, el uso indiscriminado de la ultrasonografía ha contribuido sustancialmente al diagnóstico de pequeñas masas renales que otrora pasaban desapercibidas.

Las referencias bibliográficas son escasas en lo concerniente a la historia natural de esas masas renales pequeñas. Probablemente el crecimiento anual no exceda los $0,5 \mathrm{~cm}^{2-4}$. Datos preliminares sobre el estudio de la EORTC 30904 comparando cirugía renal radical con cirugía parcial en masas renales con diámetro máximo de $4 \mathrm{~cm}$., demuestran que en este grupo, el porcentaje de lesiones benignas asciende al 15\%. Otros autores sugieren incluso un porcentaje mayor de lesiones benignas, de hasta el $22 \%$, cuando se analizan las masas renales menores de $4 \mathrm{~cm}$ de diámetro ${ }^{5}$. Reciente revisión retrospectiva de la Clínica Mayo sugiere que la proporción de tumores de bajo grado es mayor en las masas menores de $4 \mathrm{~cm}$ de diámetro $^{6}$. Diámetros tumorales de 4,5-5 cm predicen la sobrevida especifica en los registros de cáncer de Riñón de la Cleveland Clinic y de la $\mathrm{UCLA}^{7,8}$. La cirugía parcial proporciona tasas de supervivencia específica a 5 y 10 años superiores al $90 \%$ en este grupo de pacientes ${ }^{9}$.

$\mathrm{Ni}$ crecimiento ni estirpe anatomopatológica son predecibles de antemano. La CT o la punción biópsica y aspiración con aguja fina no ofrecen sensibilidad y sobre todo especificidad suficientes para determinar la naturaleza o agresividad de estas pequeñas masas ${ }^{10-12}$.

De todo lo anterior podemos concluir que asistimos a un aumento progresivo del diagnóstico de masas renales de pequeño tamaño y que el porcentaje de masas benignas o con bajo potencial biológico, en esa muestra parece ser considerable. En segundo lugar podemos afirmar que aún en ausencia de resultados a largo plazo de los estudios comparativos en curso (EORTC 30904), la nefrectomía parcial es una opción perfectamente viable con resultados extrapolables a los de la cirugía radical en casos de tumores renales de pequeño tamaño ( $\leq 4 \mathrm{~cm}$ diámetro).

Tras el diagnostico de una masa renal menor de $4 \mathrm{~cm}$., las opciones son la vigilancia expectante, la nefrectomía parcial o el uso de un tratamiento ablativo en forma de crioterapia o radiofrecuencia.

Optimizar el tratamiento de esas pequeñas masas renales tiene dos objetivos: la máxima conservación de la masa renal funcionante y la disminución de la morbilidad del tratamiento.

Si bien es cierto que la Nefrectomía parcial cumple el primero de estos propósitos, no es una cirugía exenta de complicaciones y sobre todo comporta una cicatriz (lumbotomía) con una alta morbilidad $^{13}$. La nefrectomía parcial laparoscópica, que obvia la molesta cicatriz, es una cirugía de extrema dificultad y que no está al alcance de todos los centros ${ }^{14}$.

El objetivo del presente trabajo es la revisión de la literatura sobre crioterapia en pequeñas masas renales y la descripción preliminar de nuestra experiencia en la crioterapia laparoscópica con sondas finas.

\section{CRIOTERAPIA EN MASAS RENALES}

El tratamiento ablativo de las masas renales emerge como una alternativa viable cuando se pretende control local y se desea evitar la cirugía parcial o total. Los tratamientos ablativos aúnan las ventajas del control laparoscópico (o radiológico) a las de un tratamiento mínimamente invasivo y que preserva el parénquima renal no tumoral.

Al margen de aquellos casos de diagnóstico incidental en presencia de riñón contrateral sano y en ausencia de comorbilidad, los tratamientos ablativos son de elección en determinadas situaciones clínicas en las que la ablación es preferible a la resección vg. en casos de progresivo desarrollo tumoral durante el curso de la vida como 
sería la enfermedad de Von Hippel Lindau o en pacientes con insuficiencia renal o monorrenos así como en aquellos que con limitada expectativa de vida no serían tributarios de una cirugía agresiva.

Entre los distintos métodos ablativos, crioterapia y radiofrecuencia han superado en la actualidad la fase experimental, si bien ambos se encuentran todavía en fase de desarrollo clínico. La primera, en la que tenemos experiencia, es el objeto de nuestra descripción.

\section{Principios de la Crioablación}

La crioterapia es el tratamiento del tejido mediante la formación de hielo. En 1964 Cooper concluye que temperaturas de $-20^{\circ} \mathrm{C}$ mantenidas durante un minuto causan muerte celular ${ }^{15}$. En los años siguientes se establece el papel capital que la repetición de ciclos rápidos de congelación y lentos de calentamiento poseen en la lesión celular por frío ${ }^{16}$. Durante el proceso de crioablación, la energía se extrae del tejido diana mediante una criosonda insertada en el tumor. A medida que disminuye la temperatura se produce enfriamiento y ulterior congelación del tejido con formación de una bola de hielo alrededor de la criosonda. La congelación y posterior calentamiento producen la muerte celular por diversos mecanismos. Durante el tratamiento propiamente dicho, la crioterapia produce lesión celular directa e inmediata. Tras el tratamiento acontece la lesión tisular tardía o indirecta por daño irreversible en la microvascularización.

A diferencia de lo que ocurre en la radioterapia, el mecanismo de acción de la crioterapia no depende de las características nucleares de la población tumoral sino de la exposición del tumor a un proceso letal de enfriamiento a temperatura de congelación. La supervivencia de las células depende no solamente de los ciclos de congelación y calentamiento sino de la más baja temperatura alcanzada durante el proceso y del tiempo transcurrido a temperaturas subcero. Una vez más se cumple en este procedimiento ablativo la ecuación térmica en que la cantidad de muerte celular es proporcional a la temperatura crítica y al tiempo que esta se mantiene.

Durante la crioablación, y a medida que la temperatura disminuye por debajo de $0^{\circ} \mathrm{C}$ el agua del espacio extracelular cristaliza creándose un ambiente extracelular hiperosmótico que induce la salida de agua desde la célula al espacio extracelular. Este fenómeno de deshidratación celular ocurre predominantemente entre 0 y $-20^{\circ} \mathrm{C}$. La membrana lipídica de la célula impide en esta primera fase la formación de hielo intracelular por lo que se produce un estado de imbalance osmótico entre el ambiente extra y el intracelular. Con tiempo suficiente esa hiperconcentración intracelular de electrolitos sería suficiente para destruir la célula. Posteriormente y a medida que los solutos escapan de la célula, se iguala la concentración intra y extracelular produciéndose la formación de hielo intracelular y el estallido osmótico de la célula ${ }^{17}$. Por debajo de $-20^{\circ} \mathrm{C}$ se inicia la formación de hielo intracelular. A temperaturas menores de $-40^{\circ} \mathrm{C}$ todas las células contienen cristales causantes de muerte celular irreversible. Desde la perspectiva clínica, la formación de hielo intracelular es más eficiente cuando las bajas temperaturas consistentes con el proceso se alcanzan de manera rápida.

Durante el ciclo de calentamiento posterior hasta temperaturas de $0^{\circ} \mathrm{C}$ los cristales de hielo se fusionan formando grandes cristales que rompen la membrana celular causando daño adicional. A medida que el hielo se funde, el ambiente extracelular deviene hipotónico y el exceso de agua extracelular penetra las células ya dañadas incrementando su volumen y la posibilidad de ruptura de la membrana celular ${ }^{18}$.

Por esta razón, cada uno de los ciclos de enfriamiento se sigue de un ciclo de calentamiento, al objeto de optimizar la cantidad de tejido dañado y la ablación. La mayoría de la bola de hielo experimenta bajas temperaturas que causan deshidratación celular por el mecanismo de imbalance osmótico ${ }^{19}$.

En las horas y días siguientes se produce el daño indirecto del tejido por lesión vascular. La hipoxia celular es probablemente el principal mecanismo letal de la crioablación. Estasis vascular se ha demostrado a temperaturas de $-20^{\circ} \mathrm{C}$. Durante el ciclo inicial de enfriamiento se produce vasoconstricción, disminución del flujo sanguíneo y eventual ausencia de circulación al final del congelado. Durante el calentamiento la circulación se repone con vasodilatación compensatoria. 
El daño de las células endoteliales de los pequeños vasos sanguíneos por efecto directo durante la formación de la bola de hielo comportara un aumento de la permeabilidad capilar, edema, agregación plaquetaria y fenómenos tromboembólicos endovasculares, creándose un ambiente isquémico que contribuirá a la muerte celular diferida ${ }^{18,20}$.

La repetición de este ciclo de tratamiento se asocia con una mayor y segura destrucción tisular ya que la célula dañada por un primer ciclo es más susceptible a los cambios fisicoquímicos. También los cristales intracelulares son mayores durante el segundo ciclo de congelado $^{21}$ y con mayor potencial lesivo. En cada ciclo sucesivo el enfriamiento tisular es más rápido con lo que aumenta el volumen tisular afectado $^{18}$ por lo tanto la repetición del ciclo de congelado/calentamiento incrementa la cantidad de necrosis. Varios artículos señalan que la integridad de estructuras cercanas a la bola de hielo, como el sistema colector no esta comprometida por la exposición a las bajas temperaturas criogénicas salvo en caso de punción directa ${ }^{22}$.

Los gases empleados para enfriamiento son el Argón, debido a su facilidad de manejo, y menos frecuentemente el Nitrógeno. Para calentar o descongelar se emplea el Helio.

De manera general se admite en la literatura que el congelado debe conseguirse de una manera rápida mientras que el calentamiento se realiza de manera lenta. En contra de la teoría del calentamiento pasivo preconizada por la mayoría de los clínicos, que este se consiga de manera activa o pasiva no parece influenciar la cantidad de necrosis.

Es consenso que el número mínimo de ciclos de congelado/calentamiento que deben realizarse durante el tratamiento es de dos.

Se ha demostrado experimentalmente que la criodestrucción renal produce ablación tisular completa. Dos ciclos de congelación producen mayor área de destrucción que un solo ciclo independientemente de que el proceso de calentamiento sea activo o pasivo. El tipo de calentamiento no afecta a la cantidad de tejido dañado ${ }^{23}$.

La crioablación puede conseguirse mediante el empleo de una sonda única de diámetro variable desde 2,4 $\mathrm{mm}$ hasta $5 \mathrm{~mm}$. o mediante el empleo de múltiples sondas finas (17 gauge) de $1,47 \mathrm{~mm}$ diámetro. El diámetro de la sonda única o el número de sondas finas dependerán del tamaño tumoral a tratar.

La temperatura es parámetro no solo clave sino también el mas fácil de medir durante crioterapia. El objetivo de la crioterapia es alcanzar temperaturas en el tejido a destruir iguales o por debajo de la temperatura letal. La temperatura necesaria para producir necrosis completa depende del tipo de célula y es diferente según la estirpe del órgano.

La temperatura que se ha relacionado con la necrosis celular de la célula renal en estudios "in Vitro" es la de $-19,4^{\circ} \mathrm{C}^{24,25}$. La necrosis completa se inicia a $3,1 \mathrm{~mm}$ dentro del límite ecográficamente visible de la bola de hielo. Lamentablemente los estudios no se han confirmado "in vivo" mediante escisión de la masa renal inmediatamente después de crioterapia y por lo tanto desconocemos si la temperatura testada "in Vitro" se asocia a destrucción tumoral completa. Por extensión de los estudios realizados en tejido prostático se admite que en los protocolos de tratamiento el objetivo es conseguir temperaturas de $-40^{\circ} \mathrm{C}$ o inferiores ${ }^{25}$.

Actualmente la temperatura puede monitorizarse mediante termo sensores colocados en el margen tumoral o mediante la verificación y seguimiento ecográficos de la bola de hielo. La temperatura en la periferia del tumor debe ser la necesaria para producir necrosis.

\section{Técnica quirúrgica laparoscópica}

Si bien la crioablación de las masas renales puede realizarse mediante control ecográfico, CT o RMI, describiremos a continuación la crioablación de las masas renales bajo control visual laparoscópico que es la que interesa al urólogo.

La vía de abordaje dependerá de la situación del tumor. Será transabdominal cuando el tumor se encuentre situado en la cara anterior del riñón $\mathrm{y}$ retroperitoneal en aquellos casos en que el tumor se localice en la cara posterior o en la convexidad dorsal del órgano.

Puesto que no se han demostrado lesiones del sistema colector salvo en caso de punción directa, no seria necesaria la identificación y liberación del uréter. Sin embargo y hasta mayor abun- 
dancia de datos nosotros todavía aconsejamos la liberación y rechazo medial del uréter en aquellos casos en que el tumor está en contacto directo con el uréter, esto es tumores de localización medial y en el polo inferior del riñón.

La colocación de los puertos de entrada es la utilizada de manera standard para el abordaje renal. Una vez localizado el riñón, se procede a la apertura de la fascia de Gerota y la disección del plano entre la grasa perirrenal y el parénquima renal hasta localizar el tumor. La grasa peritumoral se diseca y se envía para examen anatomopatológico. La superficie renal adyacente al tumor debe ser liberada en extensión suficiente que permita la cómoda colocación de las agujas de crioterapia y el control visual de la formación de la "bola de hielo" y del parénquima renal de aspecto sano. En el caso de abordaje transperitoneal el riñón debe liberarse del intestino $u$ otros órganos abdominales al objeto de evitar lesiones de contacto producidas por el hielo (Fig. 1).

Una vez identificada la masa tumoral, el tamaño se controla mediante ecografía intracorporal (Fig. 2). Es también esta maniobra de medición la que determinara en última instancia el número de crio-sondas a emplear en virtud del diámetro a tratar.

A continuación se procede a la práctica de la biopsia de la masa tumoral mediante pistola de punción automática y a través del trocar perpendicular a la masa o percutáneamente.

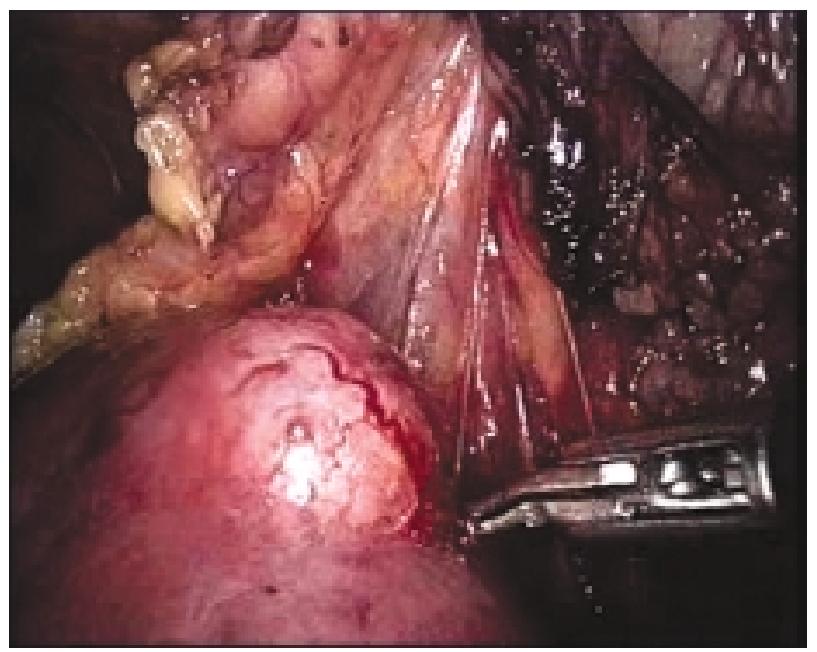

FIGURA 1. Masa en el tercio medio de la cara anterior del riñón liberada laparoscópicamente previo a la crioablación.

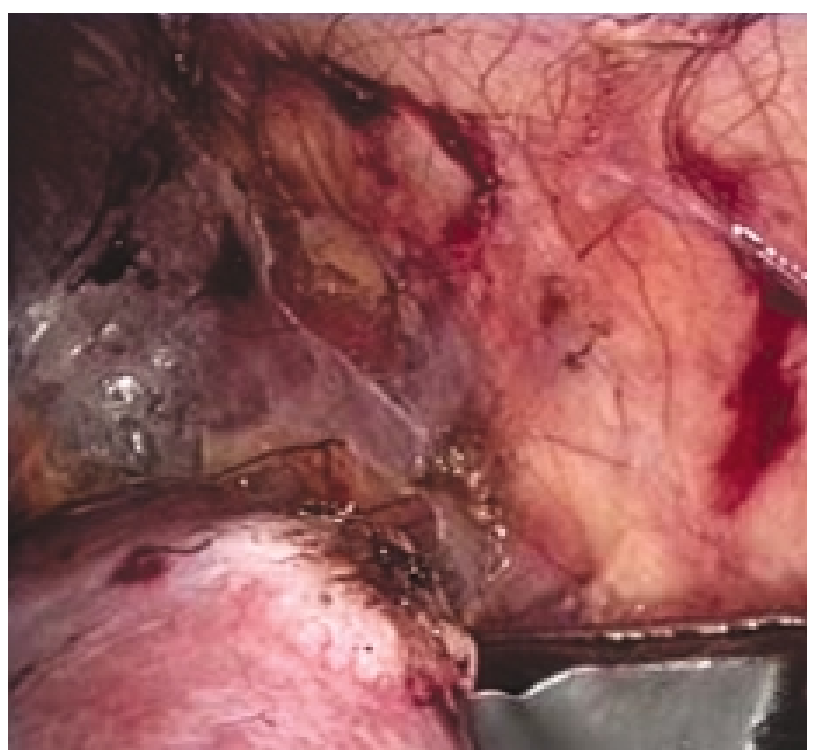

FIGURA 2. Tras la escisión de la grasa perirenal y la práctica de la biopsia, se procede a ecografia intracorporal al objeto de determinar las dimensiones precisas de la masa.

Las criosondas se colocan percutáneamente de manera perpendicular a la masa renal. La temperatura puede ser monitorizada mediante la colocación de termosondas en la zona central y en la periferia tumoral. La colocación de las termosondas asegura un correcto control de calidad y proporciona valiosa información sobre el curso del tratamiento (Fig. 3).

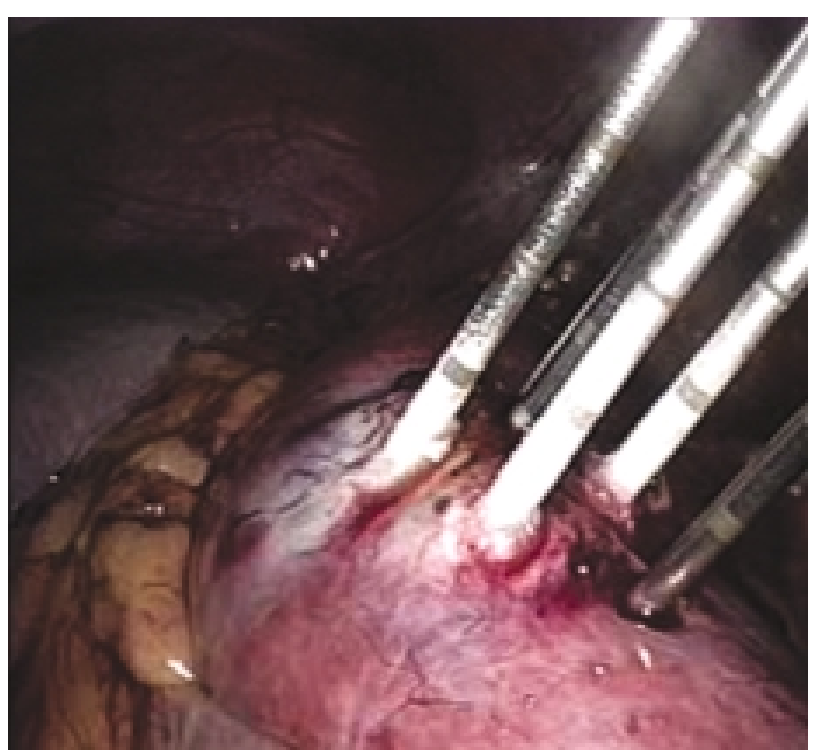

FIGURA 3. Detalle de las cuatro criosondas (17 gauge) in situ y dos termosondas adicionales, una central en la masa y otra periférica. 
Una vez colocadas las criosondas y siempre bajo control visual se procede a los dos ciclos consecutivos de congelado y calentamiento (Fig. 4). Debido al uso de gas, durante este tiempo puede aumentar la presión intracavitaria por lo que el asistente deberá abrir regularmente uno de los puertos al objeto de permitir el escape de gas. La creación de la bola de hielo se objetiva fácilmente objetivada mediante la ecografía peroperatoria intraluminal (Fig. 5).

Durante los ciclos de enfriamiento deberá irrigarse la zona cutánea por la que penetran las criosondas con suero caliente al objeto de evitar lesiones cutáneas.

Una vez finalizado el procedimiento se procede a la extracción cuidadosa, nunca brusca o antes de la descongelación, de las criosondas. Un sangrado mínimo de los sitios de punción tumoral se constata en la mayoría de los casos. En nuestra experiencia este se controla mediante corriente bipolar o la compresión mediante Surgicel durante unos minutos.

La intervención finaliza con la colocación de un drenaje en aquellos casos en que se considere necesario.

\section{Experiencia en el AMC}

Desde octubre 2003 hasta febrero 2005, 13 pacientes portadores de masas renales únicas y

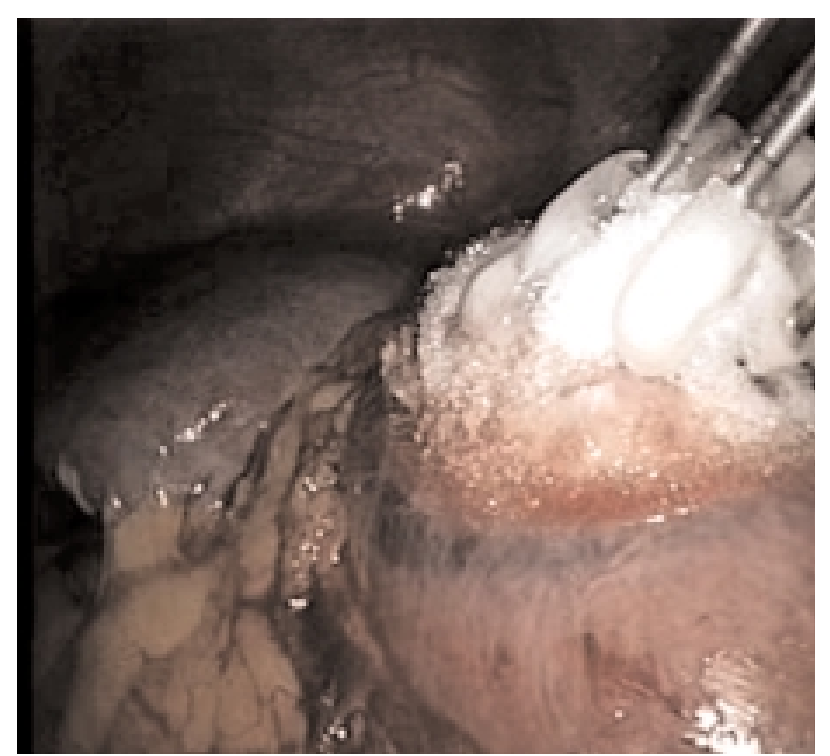

FIGURA 4. Criablación, ciclo de congelado, obsérvese la lesión periférica, en forma de hematoma.

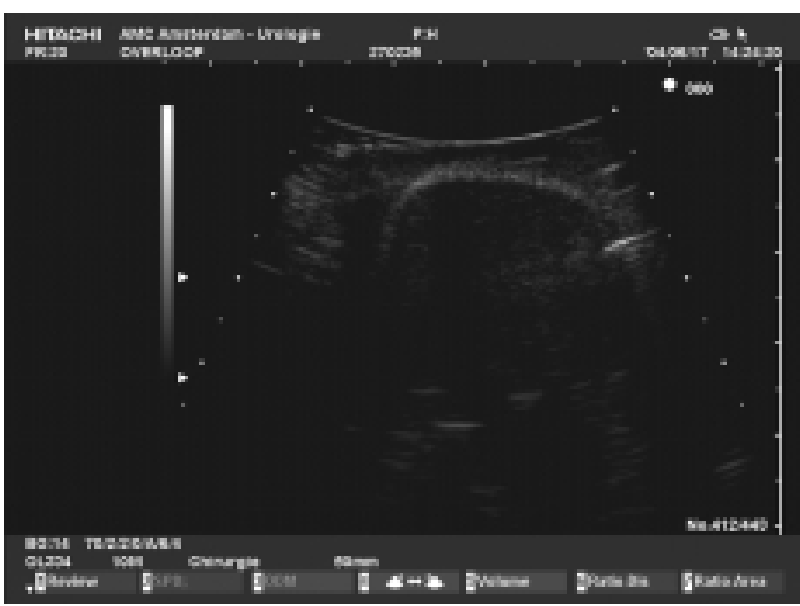

FIGURA 5. Aspecto de "Ice ball"en ecografia intracorpórea.

menores de $4 \mathrm{~cm}$. han sido tratados mediante crioablación laparoscópica según la técnica descrita con sondas ultrafinas de 1,5 mm de diámetro $\left(\right.$ Oncura $\left.{ }^{\mathrm{TM}}\right)$. Las indicaciones en nuestro Centro se especifican en la Tabla 1.

El riñón se abordó vía transperitoneal o retroperitoneal dependiendo de la situación, prefiriéndose el abordaje transperitoneal para aquellos tumores situados en la superficie anterior del riñón y el retroperitoneal para los situados en la cara posterior.

Las características demográficas y basales así como la vía de abordaje se describen en la Tabla 2.

Durante la cirugía se colocaron dos termosensores, uno de localización central en el tumor y uno periférico en el límite tumoral, al objeto de monitorizar temperaturas.

Todos los casos se completaron satisfactoriamente vía endoscópica a excepción de un paciente que presentó hipercapnia severa durante la retroperitoneoscopia y requirió de re-conversión para la colocación de las criosondas.

El tiempo medio operatorio fue de $208 \mathrm{minu}$ tos (106-397 min). El tiempo medio de exposición a temperaturas inferiores a $-20^{\circ} \mathrm{C}$ en la periferia tumoral fue de 10 minutos ( \pm 55 segundos).

Tabla 1

Indicaciones de Crioablación en masas renales

Masas renales menores de $3,5 \mathrm{~cm}$

Lesiones de situación periférica y exofítica

Lesiones de situación central identificables mediante ecografía intracorpórea 
Tabla 2

Características demográficas de la serie de crioablación del AMC

\begin{tabular}{|c|c|c|c|c|c|}
\hline & Edad / Sexo & $\varnothing$ tumor $(\mathrm{cm})$ & Situación (polo/ región) & Lado & Abordaje \\
\hline Paciente 1 & $78 / \mathrm{V}$ & 2.4 & Medio & $\mathrm{D}$ & Trans \\
\hline Paciente 2 & $77 / \mathrm{H}$ & 2.2 & Inferior & I & Trans \\
\hline Paciente 3 & $80 / V$ & 2.7 & Inferior & I & Trans \\
\hline Paciente 4 & $69 / \mathrm{V}$ & 2.4 & Superior & $\mathrm{D}$ & Trans \\
\hline Paciente 5 & $69 / \mathrm{H}$ & 1.7 & Medio & I & Retro \\
\hline Paciente 6 & $51 / \mathrm{V}$ & 1.8 & Inferior & I & Retro \\
\hline Paciente 7 & $56 / \mathrm{V}$ & 1.5 & Inferior & I & Trans \\
\hline Paciente 8 & $52 / \mathrm{H}$ & 1.3 & Medio & I & Trans \\
\hline Paciente 9 & $78 / \mathrm{H}$ & 3.0 & Superior & I & Retro \\
\hline Paciente 10 & $53 / \mathrm{V}$ & 2.1 & Medio & I & Trans \\
\hline Paciente 11 & $60 / \mathrm{V}$ & 3.2 & Inferior & $\mathrm{D}$ & Trans \\
\hline Paciente 12 & $68 / \mathrm{H}$ & 2.0 & Superior & I & Trans \\
\hline Paciente 13 & $56 / \mathrm{H}$ & 1.3 & Superior & I & Retro \\
\hline
\end{tabular}

En el postoperatorio inmediato un paciente presentó neumonía y otro infección urinaria. La estancia hospitalaria media fue de 5 días $^{3-9}$.

Durante el seguimiento se procedió a CT scan abdominal o RMI, determinación de Creatinina, enzimas hepáticas, fosfatasas alcalinas y hematología básica cada 3 meses durante el primer año y semestralmente durante el segundo año. En caso de sospecha radiológica de persistencia o recurrencia se contempla la biopsia dirigida.

Con un tiempo medio de seguimiento de $8 \mathrm{me}-$ ses (2-18 meses) todos los pacientes están vivos y sin evidencia de recurrencia tumoral radiológica.

\section{Resultados en la literatura}

Una reciente revisión de la literatura demuestra que la crioterapia es el tratamiento ablativo de las pequeñas masas renales más extensamente investigado. No existen trabajos comparativos con la cirugía convencional y la elección del control laparoscópico o el apoyo radiológico, parece depender de las características del Centro, de la disponibilidad o interés de los distintos especialistas, de la situación del tumor y de características propias del paciente.

En la mayoría de las series publicadas el número de pacientes es escaso y en general los seguimientos son cortos, lo que impide todavía juicios definitivos sobre el valor de la crioterapia.

Los resultados, sin pretensión exhaustiva se especifican en la Tabla $3^{26-34}$.

El sangrado medio peroperatorio en las series de abordaje laparoscópico oscila entre 70 y 165 $\mathrm{ml}^{26,28,32-34}$ y la estancia media hospitalaria entre 2,9 y 3,8 días $^{28,32,33}$ siendo menor de un día para el $69 \%$ de los pacientes de la serie de Gill $^{26}$.

Al margen de lo reflejado en la Tabla 3 el lector debe saber que hasta la fecha algunas series como la de la Cleveland Clinic Foundation o la de la Universidad de Jacksonville incluyen ya un número de pacientes superior a 100 , con resultados presentados preliminarmente en diferentes reuniones de expertos ${ }^{35}$.

Tabla 3

Resultados de la ablación mediante crioterapia en masas renales de pequeño tamaño

\begin{tabular}{|c|c|c|c|c|c|c|c|}
\hline & Acceso & No tumores & $\begin{array}{c}\varnothing \\
(\mathbf{c m})\end{array}$ & $\begin{array}{c}\text { Sonda } \\
(\mathbf{m m})\end{array}$ & $\begin{array}{c}\text { T-O } \\
\text { (min) }\end{array}$ & $\begin{array}{c}\text { F-U } \\
\text { meses }\end{array}$ & $\begin{array}{l}\text { Recurrencia/ } \\
\text { Persistencia }\end{array}$ \\
\hline Rukstalis, 2001 & abierto & 29 & 2,2 & $3-8$ & & 16 & 1 caso \\
\hline Lee, 2003 & laparo & 20 & 2,6 & 4,8 & 305 & 14 & 1 caso \\
\hline Nadler, 2003 & laparo & 15 & 2,15 & 4,8 & 260 & 15 & 2 casos \\
\hline Shingleton, 2004 & MRI & 111 & 3 & 3 & 79 & 30 & $8 \% *$ \\
\hline Cestari, 2004 & laparo & 37 & 2,6 & 3,2 & 194 & 20,5 & 1 caso \\
\hline Ankem, 2005 & laparo & 22 & 2,6 & $2,4-5$ & 188 & 10,4 & 0 casos \\
\hline Gill, 2005 & laparo & 60 & 2,3 & 4,8 & 180 & $>3$ años & $5,3 \% * *$ \\
\hline
\end{tabular}

* $14 \%$ re-tratamientos

**En RCC demostrado por biopsia / 5,6\% apariciones de novo. 
Las complicaciones de la crioterapia no parecen ser abundantes, ya sea por la escasa presentación o por la todavía limitación numérica de la mayoría de las series. Un reciente artículo de revisión multi-institucional en la que se incluyeron 271 pacientes tratados mediante crioterapia o radiofrecuencia ( $\mathrm{RF}$ ) (139 crioablaciones y 133 RF de los cuales 181 percutáneas y 90 laparoscópicas) procedentes de 4 instituciones demuestra una ocurrencia de complicaciones del 11\% ( $1,8 \%$ mayores y $9,2 \%$ menores) con un caso de muerte $(0,4 \%)^{36}$. La mayoría de las complicaciones $(7 \%)$ fueron directamente imputables al procedimiento ablativo. La complicación más frecuente parece ser dolor en el lugar de inserción de la/las criosondas. En bajo porcentaje complicaciones mayores como lesiones esplénicas y fractura renal han sido descritas, especialmente en caso de riñones frágiles o con antecedentes de pielonefritis $^{30}$.

\section{Seguimiento}

El objetivo de la crioablación no es la escisión de la masa tumoral sino la necrosis "in situ". Tras crioterapia la lesión permanece pero sufre cambios asociados al proceso reactivo: edema e inflamación como respuesta a la agresión y necrosis como respuesta al tratamiento. Ya que el diagnóstico de masa renal tumoral se realiza en base a las características radiológicas, esto es masa sólida ecográficamente y captante de contraste en la CT axial, estas dos exploraciones serían las idóneas para monitorizar el seguimiento. Sin embargo, la ecografía tiene un valor limitado, dado que la masa sufre una reducción de tamaño pero persiste en la mayoría de los casos.

La CT axial o la RMI por su carácter de test funcional son las adecuadas para controlar la evolución postoperatoria de la masa renal tratada mediante crioablación.

En los días inmediatos al tratamiento, la RMI demuestra una lesión de apariencia infartada con una mínima captación del contraste pero de mayor tamaño que la lesión primitiva ${ }^{33}$. Posteriormente tiene lugar una contracción gradual en el diámetro de la lesión. Resolución completa o presencia de una pequeña lesión residual parenquimatosa no captante es la norma en la mayoría de los pacientes a los tres meses de la ablación (91\% en la serie de Rukstalis) ${ }^{30}$. También en la CT se demuestra un aumento inicial del diámetro de la lesión con infiltración del tejido vecino compatible con el proceso histológico de infarto (Fig. 6). El criterio decisivo en el control de la lesión es la captación de contraste que debe ser menor de 10 unidades Hounsfield (Fig. 7).

Las recurrencias a largo plazo son escasas pero se han descrito persistencias atribuibles a insuficiente tratamiento. Recidivas o fallos del tratamiento son más frecuentes a medida que aumenta el diámetro tumoral a $\operatorname{tratar}^{28}$.

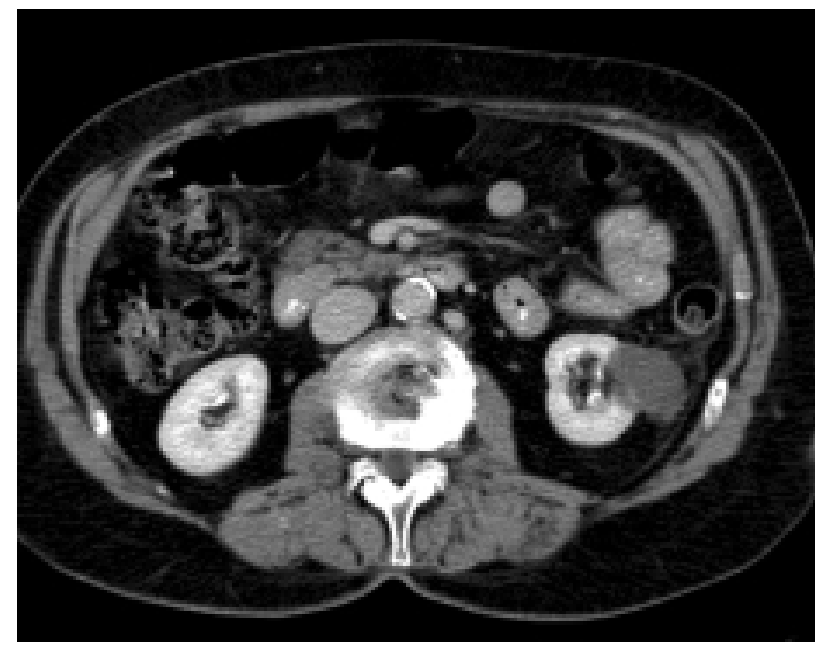

FIGURA 6. CT de masa renal, 4 semanas tras tratamiento: aumento del tamaño pero ausencia de captación de contraste.

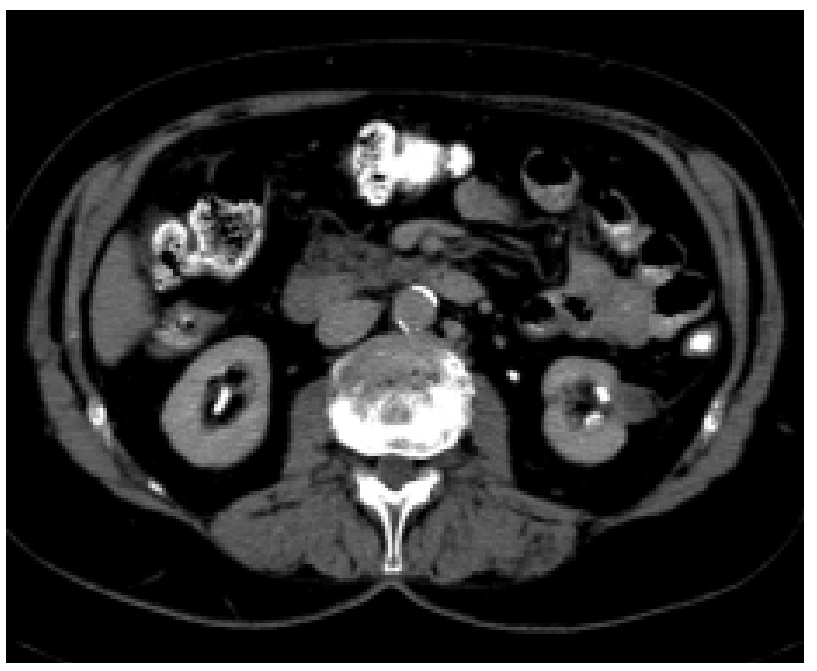

FIGURA 7. CT de la misma masa 16 semanas tras crioablación: sensible disminución de tamaño y ausencia de captación del contraste. 


\section{CONCLUSIONES}

La ablación mediante crioterapia representa un tratamiento mínimamente invasivo real en casos de masa renales de pequeño tamaño y bajo grado. Los estudios, no comparativos, llevados a cabo demuestran baja morbilidad y las recidivas a corto/medio plazo son escasas. Sin embargo, estos resultados deben todavía contemplarse con cautela, dado que por el momento es imposible determinar si son el resultado de un tratamiento idóneo $\mathrm{u}$ obedecen al esperado curso natural de la enfermedad.

\section{REFERENCIAS}

1. Hock LM, Lynch J, Balaji KC. Increasing incidence of all stages of kidney cancer in the last 2 decades in the United States: an analysis of surveillance, epidemiology and end results program data. J Urol 2002;167:57-60.

2. Wehle MJ, Thiel DD, Petrou SP, Young PR, Frank I, Karsteadt $\mathrm{N}$. Conservative management of incidental contrast-enhancing renal masses as safe alternative to invasive therapy. Urology 2004;64:49-52.

3. Kato M, Suzuki T, Suzuki Y, Terasawa Y, Sasano H, Arai Y. Natural history of small renal cell carcinoma: evaluation of growth rate, histological grade, cell proliferation and apoptosis. J Urol 2004;172:863-866.

4. Kassouf W, Aprikian AG, Lapalante M, Tanguay S. Natural history of renal masses followed expectantly. J Urol 2004,171:11113 .

5. Dechet CB, Sebo T, Farrow G, Blute ML, Engen DE, Zincke H. Prospective analysis of intraoperative frozen needle biopsy of solid renal masses in adults. J Urol 1999;162:1282-1284.

6. Frank I, Blute ML, Cheville JC, Lohse CM, Weaver AL, Zincke H. Solid renal tumors: an analysis of pathological features related to tumor size. J Urol 2003;170:2217-2220.

7. Lau WK Cheville JC, Blute ML, Weaver AL, Zincke H. Prognostic features of pathologic stage T1 renal cell carcinoma alter radical nephrectomy. Urology 2002;59:532-537.

8. Zisman A, Pantuck AJ, Wieder J, Chao DH, Dorey F, Said JW, de Kernion JB, Figlin RA, Belldegrun AS. Risk group assessment and clinical outcome algorithm to predict the natural history of patients with surgically resected renal cell carcinoma. Clin Oncol 2002;20:4559-4566.

9. Hafez KS, Fergany AF, Novick AC. Nephron sparing surgery for localized renal cell carcinoma: impact of tumor size on patient survival, tumor recurrence and TNM staging. J Urol 1999; 162:1930-1933.

10. Dechet CB, Zincke H, Sebo T, King B, Leroy AJ, Farrow GM, et al. Prospective analisis of computerized tomography and needle biopsy with permanent sectioning to determine the nature of solid renal masses in adults. J Urol 2003;169:71-74.

11. Neuzillet Y, Lechevallier E, Andre M, Daniel L, Coulange C. Accuracy and clinical role of fine needle percutaneous biopsy with computerized tomography guidance of small (less than 4.0 $\mathrm{cm})$ renal masses. J Urol 2004;171:1802-1805.

12. Rubicki FJ, Shu KM, Cibas ES, Fielding JR, van Sonneberg E, Silverman SG. Percutaneous biopsy of renal masses: sensitivity and negative predictive value stratified by clinical setting and size of the masses. AJR 2003;80:1281-1287.

13. Chatterjee S, Nam R, Fleshber N, Klotz L. Permanent flank bulge is a consequence of flank incision for radical nephrectomy in one half of patients. Urol Oncol 2004;22:36-39.

14. Guillonneau B, Abbou CC, Doublet JD, Gaston R, Janetschek G, Mandressi A, Rassweiler JJ, Vallancien G. Proposal for a "European Scoring System for laparoscopic operations in Urology". Eur Urol 2001;40:2-6.

15. Cooper IS. Cryobiology as viewed by the surgeon. Cryobiology 1964; 1:44-54.
16. Cahan W. Cryosurgery of malignant and benign tumors. Fed Proc 1965;24:S241-S248.

17. Finelli A, Rewcastle JC, Jewett MAS. Cryotherapy and radiofrequency ablation: pathophysiologic basis and laboratory studies. Curr Opin Urol 2003;13:187-191.

18. Theodorescu D. Cancer cryotherapy: evolution and biology. Rev Urol 2004;6(suppl 4):S9-S19.

19. Bischof JC, Smith D, Pazhayannur PV et al. Cryosurgery of dumming AT-1 rat prostate tumor: thermal, biophysical and viability response at the cellular and tissue level. Cryobiology 1997;34:42-69.

20. Baust JM, Van Buskirk RG, Baust JG. Cell viability imporves following inhibition of cryopreservation-induced apoptosis. In Vitro Cell Dev Biol Anim 2000;36:262-270.

21. Whittaker DK. Repeat freeze cycles in cryosurgery of oral tisues. Br Dent J 1975;139:459-465.

22. Shingleton WB, Farabaugh P, Hughson M, Sewell P. Effects of cryoablation on short term development of urinary fistulas in the porcine kidney. J Endourol 2003;17:37-40.

23. Woolley ML, Schulsinger DA, Durand DB, Zelster IS, Waltzer WC. Effect of freezing parameters (freeze cycle and thawn process) on tissue destruction following renal cryoablation. J Endourol 2002;16:519-522.

24. Rupp CC, Hoffmann NE, Schmidlin FR, Swandlund DJ, Bischof JC, Coan JE.Cryosurgical changes in the porcine kidney: histologic analysis with thermal correlation. Cryobiology 2002;45:167-182.

25. Chosy SG, Nakada SY, Lee jr FT, Warner TF. Monitoring renal cryosurgery: predictors of tissue necrosis in swine. J Urol 1998; 159:1370-1374.

26. Gill IS, Novick AC, Meraney AM, Chen RN, Hobart MG, Sung GT, H, et al. Laparoscopic cryoablation in 32 patients. Urology 2000;56:748-753.

27. Lee DI, McGinnis DE, Feld R, Strup SE. Retroperitoneal laparoscopic cryoablation of small renal tumors: intermediate results. Urology 2003;61:83-88.

28. Nadler RB, Kim SC, Rubenstein JN, Yap RL, Campbell SC, User HM. Laparoscopic renal cryosurgery: the Northwestern experience. J Urol 2003;170:1121-1125.

29. Rodríguez R, Chan DY, Bishoff JT, Chen RB, Kavoussi LR, Chota MA, et al. Renal ablativo cryosurgery in selected patients with peripheral renal masses. Urology 2000;55:25-30.

30. Rukstalis DB, Khorsandi M, Garcia FU, Hoening DM, Cohen JK. Clinical experience with open renal cryoablation. Urology 2001;57:34-39.

31. Shingleton WB, Sewell PE. Percutaneous renal tumor cryoablation: results in the first 90 patients. J Urol 2004;171 abstr. $1751 ; 463$.

32. Colon I, Fuchs GJ. Early experience with the laparoscopic cryoablation in patients with small renal tumors and severe comorbidity. J Endouro 2003;17:415-423.

33. Cestari A, Guazzoni G, dell'Acqua V, Nava L, Cardone G, Balconi G, et al. Laparoscopic cryoablation of solid renal masses: intermediate term follow-up. J Urol 2004;172:1267-1270.

34. Bachmann A, Sulser T, Jayet Ch, Wyler S, Ruszat R, Reich O, et al. Retroperitoneoscopy-assisted Cryoablation of Renal Tumors using multiple $1.5 \mathrm{~mm}$ ultrathin cryoprobes: a preliminary report. Eur Urol 2005;47:474-479.

35. Hasan W, Gill IS, Spaliviero M, Desai MM, Kaouk J, Remer EM, et al. Renal cryoablation: 4 years follow-up. J Urol 2004;171 abstr 1657;438.

36. Johnson DB, Solomon SB, Su LM, Matsumoto ED, Kavoussi LR, Nakada S, et al. Defining the complications of cryoablation and radio frequency ablation of small renal tumors: a multiinstitutional review. J Urol 2004;172:874-877.

Dra. M.P. Laguna

Department of Urology

AMC, University of Âmsterdam. P.O.Box 22700

1100 DE Amsterdam

The Netherlands

e-mail: m.p.lagunapes@amc.uva.nl

(Tabajo recibido el 13 mayo de 2005) 